\title{
A pilot study of ezetimibe vs. atorvastatin for improving peripheral microvascular endothelial function in stable patients with type 2 diabetes mellitus
}

Seigo Sugiyama ${ }^{1,2,3^{*}{ }^{+}}$, Hideaki Jinnouchi ${ }^{1,2,4 \dagger}$, Kunio Hieshima ${ }^{1}$, Noboru Kurinami ${ }^{1}$, Tomoko Suzuki ${ }^{1}$, Fumio Miyamoto ${ }^{1}$, Keizo Kajiwara ${ }^{1,2}$, Kunihiko Matsui ${ }^{5}$ and Tomio Jinnouchi ${ }^{1,2}$

\begin{abstract}
Background: Elevated cholesterol in type 2 diabetes mellitus (DM) can cause endothelial dysfunction. An effective clinical therapy to improve endothelial dysfunction remains to be established. Different cardiovascular actions between treatments for the inhibition of cholesterol absorption and the suppression of cholesterol synthesis for achieving improvement in endothelial function are unknown in DM.

Methods: Stable patients with type 2 DM and mildly elevated low-density lipoprotein cholesterol were enrolled. We evaluated peripheral microvascular endothelial function using reactive hyperemia peripheral arterial tonometry (RH-PAT) examination and calculated a natural logarithmic transformed value for the RH-PAT index (LnRHI). We randomly assigned 33 patients to each monotherapy: cholesterol synthesis suppression using atorvastatin ( $5 \mathrm{mg} /$ day, $n=16)$ or cholesterol absorption inhibition using ezetimibe (10 mg/day, $n=17)$. Patients were prospectively followed for 6 months. Serum lipids and LnRHI were repeatedly examined before and after each therapy.

Results: LDL significantly decreased in both groups, but the percent changes of LDL showed a greater decrease in the atorvastatin group compared with the ezetimibe group $(-34.5 \pm 7.8 \%$ vs. $-21.9 \pm 9.6 \%, p<0.01)$. Serum levels of non-esterified free fatty acids (NEFA) significantly decreased in the ezetimibe group but not in the atorvastatin group (ezetimibe group: $561.1 \pm 236.8$ to $429.7 \pm 195.9, p<0.01$; atorvastatin group: $538.8 \pm 319.5$ to $520.2 \pm 227.3, p=0.75)$. The percent decrease in NEFA was significantly greater in the ezetimibe group compared with the atorvastatin group $(-19.9 \pm 27.4 \%$ vs. $11.3 \pm 44.1 \%, p<0.05)$. LnRHI showed a significant increase in the ezetimibe group but not in the atorvastatin group (ezetimibe group: $0.471 \pm 0.157$ to $0.678 \pm$ $0.187, p<0.01$; atorvastatin group: $0.552 \pm 0.084$ to $0.558 \pm 0.202, p=0.64)$. The percent changes in LnRHI were significantly greater in the ezetimibe group compared with the atorvastatin group $(63.3 \pm 89.2 \%$ vs. $7.4 \pm 41.2 \%$, $\mathrm{p}<0.05)$.
\end{abstract}

Conclusions: In patients with type 2 DM, ezetimibe monotherapy significantly reduced LDL and NEFA, and improved peripheral microvascular endothelial dysfunction. Ezetimibe could potentially exhibit beneficial effects on lipid disorders and microvascular endothelial dysfunction in DM.

Keywords: Diabetes, Ezetimibe, Statins, Cholesterol absorption, Endothelial function, Microvasculature, Free fatty acid

\footnotetext{
* Correspondence: ssugiyam@kumamoto-u.ac.jp

${ }^{\dagger}$ Equal contributors

${ }^{1}$ Diabetes Care Center, Jinnouchi Hospital, 6-2-3 Kuhonji, Chuo-ku,

Kumamoto 862-0976, Japan

2Diabetes Care Center, Cardiovascular Division, Jinnouchi Hospital, 6-2-3

Kuhonji, Chuo-ku, Kumamoto 862-0976, Japan

Full list of author information is available at the end of the article
} 


\section{Background}

Type 2 diabetes mellitus (DM) is a high-risk clinical condition for cardiovascular disease [1]. Treatment strategies that may exhibit favorable effects on cardiovascular function are thought to have additionally beneficial clinical value in DM. Vascular endothelial dysfunction is a significant and independent predictor of future cardiovascular events [2], and the presence of DM and elevated low-density lipoprotein cholesterol (LDL) are recognized as important pathological conditions leading to endothelial dysfunction in clinical practice [3]. In patients with endothelial dysfunction, it has been reported that improvement in endothelial function with optimal medical treatments successfully improved cardiovascular prognosis in patients with coronary artery disease [4]; however, an effective treatment strategy to improve endothelial dysfunction is still uncertain in DM [3,5]. Increased attention has been focused on investigating and developing practical strategies for improving vascular endothelial function clinically [3]. Reactive hyperemia peripheral arterial tonometry (RH-PAT) is a simple, easy, non-invasive, and reproducible physiological examination for testing peripheral microvascular endothelial function in the fingertip [6,7]. We previously reported the clinical utility and significance of RH-PAT testing in terms of prognostic value $[8,9]$, and on the interventional effects in patients with risk factors for cardiovascular disease [10,11].

LDL-lowering therapy is a promising and effective strategy for preventing atherothrombotic events in highrisk patients, and has been established as effective in DM [12]. There are two strategies to reduce LDL in clinical practice [1]: (1) suppression of endogenous cholesterol synthesis in the liver by statins; and (2) inhibition of cholesterol absorption in the intestine through blocking Niemann-Pick C1-Like 1 (NPC1L1) using ezetimibe [2]. Statins are generally better at lowering LDL than ezetimibe, but the clinical difference of both on cardiovascular function is not well investigated in DM.

The benefits of statins for patients with DM have been established in large-scale clinical trials [3]. However, there is a recent concern that statins may have an adverse effect on glucose metabolism [4]. Thus, re-evaluation of the comprehensive systemic benefits of LDL-lowering therapy in DM requires careful consideration and is of clinical importance [1]. Ezetimibe has been shown to reduce LDL [2] and ameliorate postprandial dyslipidemia [5] without adversely affecting glucose metabolism [1,6,7]. This suggests its potential appropriate usefulness for improving cardiovascular function and in pathological conditions in DM [8,9].

We hypothesized that ezetimibe monotherapy, as compared with atorvastatin monotherapy, as the LDL-lowering therapy could significantly improve microvascular endothelial dysfunction in patients with DM. To test this hypothesis, we prospectively enrolled stable patients with type 2 DM and mildly elevated LDL. Participants were randomly assigned to either ezetimibe or atorvastatin monotherapy and were studied for 6 months for the effects of the treatments on peripheral microvascular endothelial function assessed by RH-PAT examination.

\section{Methods}

\section{Study population and study protocol}

This was a single center, prospective, open-label, randomized, two-arm clinical trial to investigate the interand intra-group differences of the two strategies for LDL-lowering therapy on peripheral microvascular endothelial function in stable patients with type $2 \mathrm{DM}$. We primarily recruited lipid-lowering medication naïve patients with type $2 \mathrm{DM}$ and mildly elevated LDL (LDL >120 mg/dL) through the outpatient clinic at Jinnouchi Hospital in Japan. We excluded patients with unstable conditions, coronary artery disease, stroke, cancer, active inflammation, autoimmune disease, lung disease, severe liver disease, and end-stage renal dysfunction. Patients were randomly allocated by using permuted-block randomization of six to either the ezetimibe group (10 mg/day) as the cholesterol absorption inhibition therapy or the atorvastatin group (5 mg/day) as the cholesterol synthesis suppression therapy for 6 months. We repeatedly measured serum lipid concentrations and peripheral microvascular endothelial function before and after each therapy at the outpatient clinic at Jinnouchi Hospital. We did not change the anti-diabetic medication during the study period.

This study was conducted in accordance with the principles contained in the Declaration of Helsinki. The study protocol was approved by the Human Ethics Review Committee of Jinnouchi Hospital. Signed consent was obtained from each participant.

\section{Assessment of peripheral microvascular endothelial function}

The principles and details of RH-PAT examination have been described previously $[10,11]$. Briefly, we volumetrically evaluated peripheral microvascular endothelial function in the fingertip by RH-PAT using the EndoPAT2000 device (Itamar Medical, Israel). Measurements were taken when patients were in a stable condition and in the fasting state in the early morning before taking their medication. Patients were examined on a bed in the supine position after at least $10 \mathrm{~min}$ of rest in a temperature- and light-controlled environment. A blood pressure cuff was placed on the upper arm to be studied, while the contra-lateral arm served as a control. PAT probes were placed on one finger of each hand. After an equilibration period, baseline pulse volume amplitude 
was measured for each fingertip for $5 \mathrm{~min}$. The cuff was inflated to $60 \mathrm{mmHg}$ above systolic pressure or $200 \mathrm{mmHg}$ for $5 \mathrm{~min}$ before being deflated to induce reactive hyperemia. After cuff deflation, PAT recordings were made for $10 \mathrm{~min}$ and RH-PAT data were automatically analyzed in real time using computer software (Endo-PAT2000 software, version 3.0.4). The RH-PAT index (RHI) reflects the extent of reactive hyperemia and was calculated as the ratio of the average amplitude of the PAT signal over $1 \mathrm{~min}$ starting $1.5 \mathrm{~min}$ after cuff deflation (control arm, A; occluded arm, C) divided by the average amplitude of the PAT signal in the $2.5 \mathrm{~min}$ before cuff inflation (baseline: control arm, B; occluded arm, D) according to the equation (C/D)/(A/B) [10]. We calculated the natural logarithmic transformation (Ln) of the RHI values, the LnRHI [12]. We were able to minimize intra- and inter-observer variability as $\mathrm{RH}$ PAT measurements were analyzed with a computerized and automated algorithm in an operator-independent manner [13]. Previous studies have demonstrated that RH-PAT technology has excellent reproducibility $[13,14]$.

\section{Blood tests}

Fasting blood samples were collected from the antecubital vein in the morning before the therapies and after the 6 months of treatment with ezetimibe or atorvastatin. Blood analyses were conducted at the hospital laboratory for the measurement of blood glucose, hemoglobin A1c (HbA1c), cholesterol, and triglyceride. Blood lathosterol, campesterol, and non-esterified free fatty acid (NEFA) were measured by SRL Corp., Tokyo, Japan. Serum total cholesterol, triglyceride and NEFA were measured by enzymatic methods, and high-density lipoprotein (HDL) and LDL were measured by direct methods using commercial reagents (Sekisui Medical Co. Ltd., Tokyo). Fasting plasma glucose (FPG) was measured by the hexokinase method and $\mathrm{HbA} 1 \mathrm{c}$ was measured by the latex agglutination immunoassay. Campesterol and lathosterol were determined by gas chromatography.

\section{Statistical analysis}

Based on our preliminary examination at our hospital, power analysis indicated that an enrollment of $30 \mathrm{pa}-$ tients was required to detect a mean difference in percent change in LnRHI $20 \pm 12 \%$ in the ezetimibe group and $10 \pm 6 \%$ in the atorvastatin group, with a power of $80 \%$ and a two-sided alpha of 0.05 . The results of the normally distributed continuous variables (determined by the Shapiro-Wilk test) are expressed as mean (standard deviation), while those of the continuous variables with skewed distributions are expressed as median values (interquartile range). Differences in baseline characteristics of the two groups were analyzed using the
Student's $t$-test, the Mann-Whitney $U$ test or the Fisher's exact test for categorical data, as appropriate. Either the paired Student's $t$-test or the Wilcoxon test was used to analyze the effect of each treatment in intragroup analysis. The percent changes in LnRHI between the ezetimibe group and the atorvastatin group were further evaluated by analysis of variance (ANOVA) with adjustment for age, gender, and body mass index (BMI). Logistic regression analysis was used to evaluate the association between improvement in endothelial function (LnRHI increase $>40 \%$, the highest tertile) and baseline clinical variables, including age, gender, BMI, HbA1c, FPG, lipid parameters, medications, and LDL-lowering therapy allocation (ezetimibe or atorvastatin). Associations between groups and all other parameters were analyzed first by univariate logistic regression analysis, followed by multivariate logistic regression analysis using the forced inclusion model, and the Hosmer-Lemeshow goodness-of-fit statistic was calculated. To determine the relationship between changes in various clinical parameters and percent changes in LnRHI, correlations between variables of interest were analyzed using Pearson's correlation coefficient. A p-value $<0.05$ was considered statistically significant. Statistical analyses were performed using the Statistical Package for Social Sciences, version 19 (SPSS Inc., IBM, Tokyo, Japan) and SAS, version 9.4 (SAS Institute Inc., Cary, NC, USA).

\section{Results}

\section{Baseline clinical characteristics}

The study comprised 33 stable Japanese patients with type $2 \mathrm{DM}$ and mildly elevated LDL (LDL: $142 \pm$ $21.7 \mathrm{mg} / \mathrm{dL}$ ). Clinical baseline characteristics of patients and each group are shown in Table 1. Baseline characteristics of patients in the ezetimibe group were similar to those of the atorvastatin group. Baseline levels of BMI, HbA1c, FPG, total cholesterol, LDL, HDL, triglyceride, and NEFA were not significantly different between groups. There was no significant difference in peripheral microvascular endothelial function assessed by LnRHI values in the RH-PAT examination between groups at baseline (Table 2).

\section{Changes in HbA1c, FPG, and blood lipid parameters}

All patients presented with non-significant changes in HbA1c ( $7.1 \pm 0.9 \%$ to $7.1 \pm 0.9 \%, \mathrm{p}=0.98)$ and FPG $(133.7 \pm$ 21.2 to $136.4 \pm 26.4 \mathrm{mg} / \mathrm{dL}, \mathrm{p}=0.47$ ). Intra-group changes in HbA1c and FPG were not statistically significant in both groups during therapies. As shown in Table 2, total cholesterol and LDL significantly decreased in both groups, but decreases in total cholesterol and LDL were significantly greater in the atorvastatin group than in the ezetimibe group (total cholesterol: $-22.8 \pm 6.1 \%$ vs. $-17.1 \pm 9.3 \%, \mathrm{p}<0.05$; LDL: $\quad-34.5 \pm 7.8 \%$ vs. $\quad-21.9 \pm 9.6 \%, \quad \mathrm{p}<0.01)$. NEFA 
Table 1 Baseline clinical characteristics

\begin{tabular}{|c|c|c|c|}
\hline & Ezetimibe $(n=17)$ & Atorvastatin $(n=16)$ & p-value \\
\hline Age (years) & $64.2 \pm 9.8$ & $65.0 \pm 8.0$ & 0.81 \\
\hline Sex, male (\%) & $7(41.2 \%)$ & $8(50.0 \%)$ & 0.73 \\
\hline Body mass index $\left(\mathrm{kg} / \mathrm{m}^{2}\right)$ & $22.5 \pm 2.4$ & $23.3 \pm 3.5$ & 0.45 \\
\hline Hypertension (\%) & $9(56.3 \%)$ & $9(52.9 \%)$ & 1.00 \\
\hline Current smoking (\%) & $4(23.5 \%)$ & $4(25.0 \%)$ & 1.00 \\
\hline Hemoglobin A1c (\%) & $7.3 \pm 1.1$ & $6.9 \pm 0.6$ & 0.18 \\
\hline Fasting plasma glucose (mg/dL) & $135.8 \pm 21.5$ & $131.4 \pm 21.4$ & 0.57 \\
\hline Duration of diabetes (yeas) & $13.6 \pm 8.1$ & $11.9 \pm 9.3$ & 0.58 \\
\hline Total cholesterol (mg/dL) & $237.5 \pm 25.8$ & $226.6 \pm 29.5$ & 0.27 \\
\hline LDL cholesterol (ng/mL) & $148.8 \pm 22.1$ & $135.6 \pm 19.7$ & 0.08 \\
\hline HDL cholesterol (mg/dL) & $57.0 \pm 11.3$ & $54.9 \pm 19.0$ & 0.71 \\
\hline Triglyceride (mg/dL) & $112.0(100.0-192.5)$ & $134.5(76.3-229.3)$ & 0.83 \\
\hline Anti-diabetic medicines & - & - & - \\
\hline Sulfonylureas (\%) & $2(11.8 \%)$ & $5(31.5 \%)$ & 0.23 \\
\hline Metformin (\%) & $5(29.4 \%)$ & $4(25.0 \%)$ & 1.00 \\
\hline Alpha-glucosidase inhibitor (\%) & $5(29.4 \%)$ & $6(37.5 \%)$ & 0.72 \\
\hline Thiazolidinedione (\%) & $1(5.9 \%)$ & $2(12.5 \%)$ & 0.60 \\
\hline Glinide (\%) & $0(0 \%)$ & $1(6.3 \%)$ & 0.49 \\
\hline DPP-4 inhibitor (\%) & $1(5.9 \%)$ & $2(12.5 \%)$ & 0.60 \\
\hline Insulin (\%) & $8(47.1 \%)$ & $3(18.8 \%)$ & 0.14 \\
\hline Any anti-diabetic medications (\%) & $14(82.3 \%)$ & $14(87.5 \%)$ & 0.53 \\
\hline $\mathrm{LnRHI}$ & $0.471 \pm 0.157$ & $0.552 \pm 0.084$ & 0.076 \\
\hline
\end{tabular}

LDL: low-density lipoprotein, HDL: high-density lipoprotein, DPP-4: dipeptidyl peptidase-4, LnRHI: natural logarithmic transformed value of reactive hyperemia peripheral tonometry index.

significantly decreased in the ezetimibe group but not in the atorvastatin group (Table 2). The percent decrease in NEFA was significantly greater in the ezetimibe group compared with the atorvastatin group $(-19.9 \pm 27.4 \%$ vs. $11.3 \pm 44.1 \%$, $\mathrm{p}<0.05$; Table 2 and Figure 1). Campesterol, as the cholesterol absorption marker, significantly decreased in the ezetimibe group but not in the atorvastatin group. Lathosterol, as the cholesterol synthesis marker, significantly decreased in the atorvastatin group and increased in the ezetimibe group. The lathosterol to campesterol ratio, as the comprehensive indicator of cholesterol synthesis and absorption balance, exhibited a significant increase in the ezetimibe group and a significant decrease in the atorvastatin group. The percent change in the lathosterol to campesterol ratio was significantly different between the two groups (ezetimibe group: $239.3 \pm 38.5 \%$, atorvastatin-group: $-39.2 \pm$ $38.5 \%, \mathrm{p}<0.001$; Table 2 and Figure 2).

\section{Changes in peripheral microvascular endothelial function assessed by LnRHI}

During the 6-month treatments, microvascular endothelial function assessed by LnRHI significantly improved after LDL-lowering therapy in all patients (LnRHI: $0.510 \pm$ 0.131 to $0.630 \pm 0.198, p=0.01$ ). Ezetimibe monotherapy but not atorvastatin monotherapy exhibited a significant intra-group improvement in peripheral microvascular endothelial function assessed by LnRHI (ezetimibe group: $0.471 \pm 0.157$ to $0.678 \pm 0.187, \mathrm{p}<0.01$; atorvastatin group: $0.552 \pm 0.084$ to $0.558 \pm 0.202, \mathrm{p}=0.64$; Table 2). Regarding inter-group comparisons, the percent changes in LnRHI were significantly greater in the ezetimibe group compared with the atorvastatin group ( $\mathrm{p}=0.028$; Table 2 and Figure 3 ). After using ANOVA with adjustment for age, gender, and BMI, the percent changes in LnRHI were significantly greater in the ezetimibe group compared with the atorvastatin group (ANOVA, $\mathrm{p}=0.041$ ).

Univariate logistic regression analysis for various clinical factors demonstrated that only ezetimibe therapy significantly correlated with improvements in peripheral microvascular endothelial function defined as the highest tertile of the percent changes in LnRHI (Table 3, odds ratio: 4.88, 95\% confidence interval: 1.01-23.57, $\mathrm{p}=0.049$ ). Forced inclusion multivariate logistic regression analysis with age, gender, and the 
Table 2 Changes in diabetes and lipid parameters and LnRHI

\begin{tabular}{|c|c|c|c|c|c|c|c|c|}
\hline & \multicolumn{3}{|c|}{ Ezetimibe $(n=17)$} & \multirow[t]{2}{*}{$p$-value } & \multicolumn{3}{|c|}{ Atorvastatin $(n=16)$} & \multirow[t]{2}{*}{$p-$ value } \\
\hline & Baseline & & 6 months & & Baseline & & 6 months & \\
\hline Hemoglobin A1c (\%) & $7.3 \pm 1.1$ & & $7.5 \pm 0.8$ & 0.307 & $6.9 \pm 0.6$ & & $6.7 \pm 0.7$ & 0.062 \\
\hline$\%$ change $(\%)$ & & $3.9 \pm 13.3$ & & & & $-0.3 \pm 2.1$ & & \\
\hline Fasting plasma glucose (mg/dL) & $135.8 \pm 21.5$ & & $135.8 \pm 27.9$ & 0.581 & $131.4 \pm 21.4$ & & $133.9 \pm 25.5$ & 0.651 \\
\hline$\%$ change $(\%)$ & & $2.6 \pm 15.3$ & & & & $2.8 \pm 7.8$ & & \\
\hline Total cholesterol (mg/dL) & $237.5 \pm 25.8$ & & $195.7 \pm 21.6$ & $<0.001$ & $226.6 \pm 29.5$ & & $174.4 \pm 22.2$ & $<0.001$ \\
\hline$\%$ change $(\%)$ & & $-17.1 \pm 9.3 \neq$ & & & & $-22.8 \pm 6.1 \neq$ & & \\
\hline LDL cholesterol (mg/dL) & $148.8 \pm 22.1$ & & $115.5 \pm 17.9$ & $<0.001$ & $135.6 \pm 19.7$ & & $88.9 \pm 17.5$ & $<0.001$ \\
\hline$\%$ change (\%) & & $-21.9 \pm 9.6^{*}$ & & & & $-34.5 \pm 7.8^{*}$ & & \\
\hline HDL cholesterol (mg/dL) & $57.0 \pm 11.3$ & & $54.9 \pm 12.6$ & 0.31 & $54.9 \pm 19.0$ & & $58.0 \pm 18.5$ & 0.035 \\
\hline$\%$ change (\%) & & $-3.4 \pm 12.1 \neq$ & & & & $6.5 \pm 10.5 \neq$ & & \\
\hline Triglyceride (mg/dL) & $\begin{array}{l}112.0 \\
(100.0-192.5)\end{array}$ & & $\begin{array}{l}94.0 \\
(80.0-171.0)\end{array}$ & 0.18 & $\begin{array}{l}134.5 \\
(76.3-229.3)\end{array}$ & & $\begin{array}{l}105 \\
(67.0-188.3)\end{array}$ & 0.12 \\
\hline$\%$ change $(\%)$ & & $-8.9 \pm 28.1$ & & & & $-7.0 \pm 45.0$ & & \\
\hline NEFA (mg/dL) & $561.1 \pm 236.8$ & & $429.7 \pm 195.9$ & 0.009 & $538.8 \pm 319.5$ & & $520.2 \pm 227.3$ & 0.746 \\
\hline$\%$ change (\%) & & $-19.9 \pm 27.4 \neq$ & & & & $11.3 \pm 44.1 \neq$ & & \\
\hline Lathosterol $(\mu \mathrm{g} / \mathrm{mL})$ & $3.30 \pm 1.45$ & & $4.60 \pm 1.96$ & 0.004 & $3.44 \pm 1.52$ & & $1.57 \pm 0.61$ & $<0.001$ \\
\hline$\%$ change (\%) & & $51.2 \pm 52.2^{*}$ & & & & $-42.0 \pm 49.7^{*}$ & & \\
\hline Campesterol ( $\mu \mathrm{g} / \mathrm{mL})$ & $6.14 \pm 2.74$ & & $2.69 \pm 0.72$ & $<0.001$ & $6.56 \pm 1.53$ & & $6.21 \pm 1.86$ & 0.217 \\
\hline$\%$ change $(\%)$ & & $-51.0 \pm 17.7^{*}$ & & & & $-6.5 \pm 23.3^{*}$ & & \\
\hline Lathosterol/campesterol & $\begin{array}{l}0.457 \\
(0.337-1.006)\end{array}$ & & $\begin{array}{l}1.428 \\
(1.189-2.303)\end{array}$ & $<0.001$ & $\begin{array}{l}0.593 \\
(0.282-0.678)\end{array}$ & & $\begin{array}{l}0.276 \\
(0.205-0.311)\end{array}$ & 0.002 \\
\hline$\%$ change (\%) & & $239.3 \pm 38.5^{*}$ & & & & $-39.2 \pm 38.5^{*}$ & & \\
\hline LnRHI & $0.471 \pm 0.157$ & & $0.678 \pm 0.187$ & 0.007 & $0.552 \pm 0.084$ & & $0.558 \pm 0.202$ & 0.645 \\
\hline$\%$ change $(\%)$ & & $63.3 \pm 89.2 \neq$ & & & & $7.4 \pm 41.2 \neq$ & & \\
\hline
\end{tabular}

LDL: low-density lipoprotein, HDL: high-density lipoprotein, NEFA: non-esterified fatty acid, LnRHI: natural logarithmic transformed value of reactive hyperemia peripheral tonometry index. ${ }^{*} p<0.001, \neq p<0.05$; ezetimibe vs. atorvastatin.

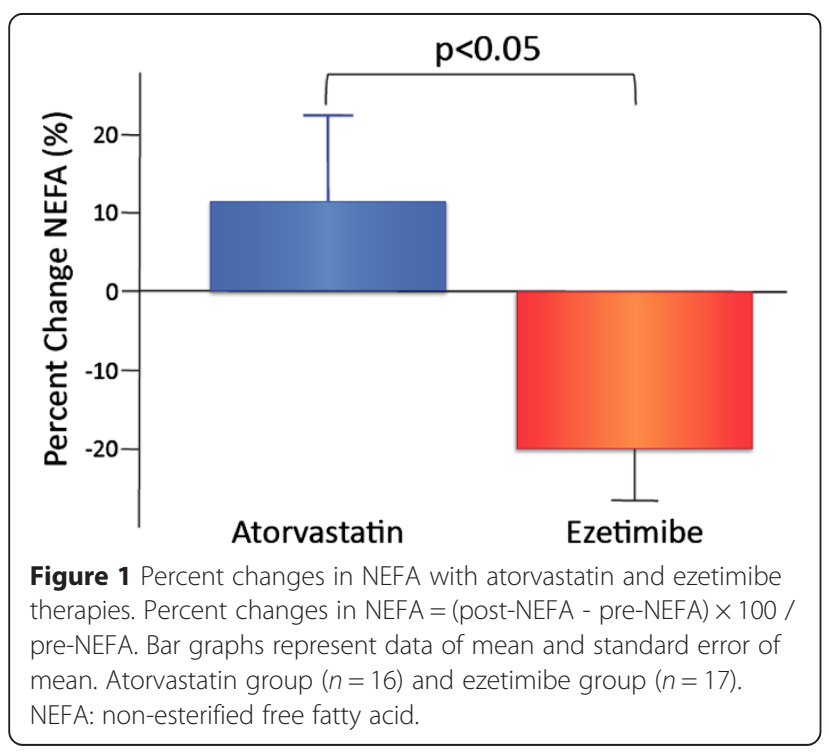

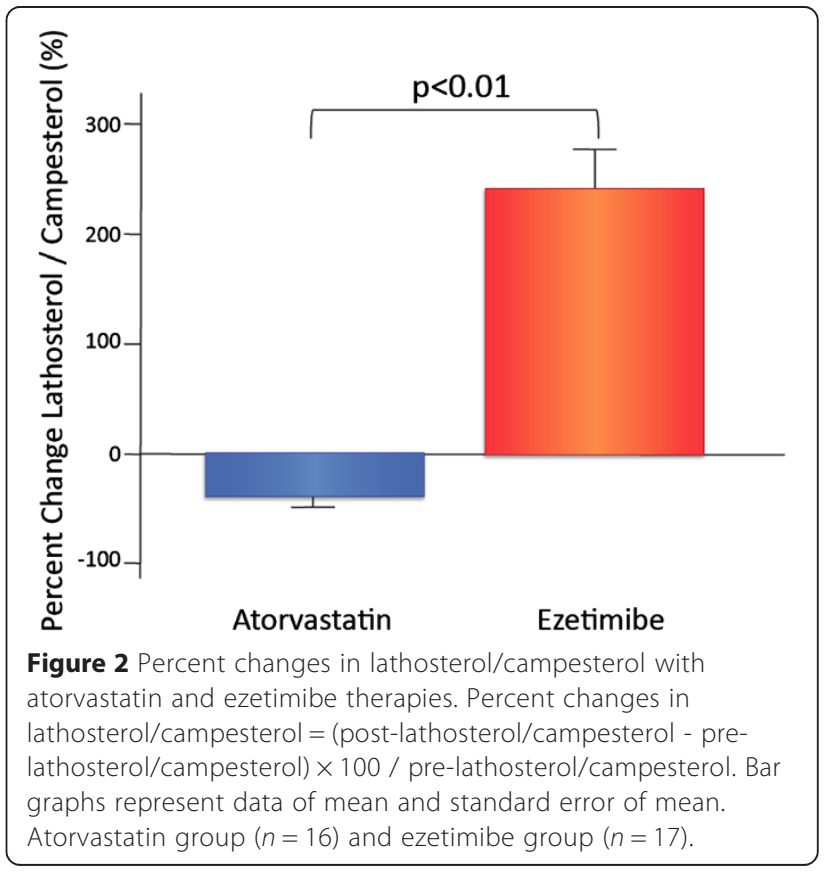




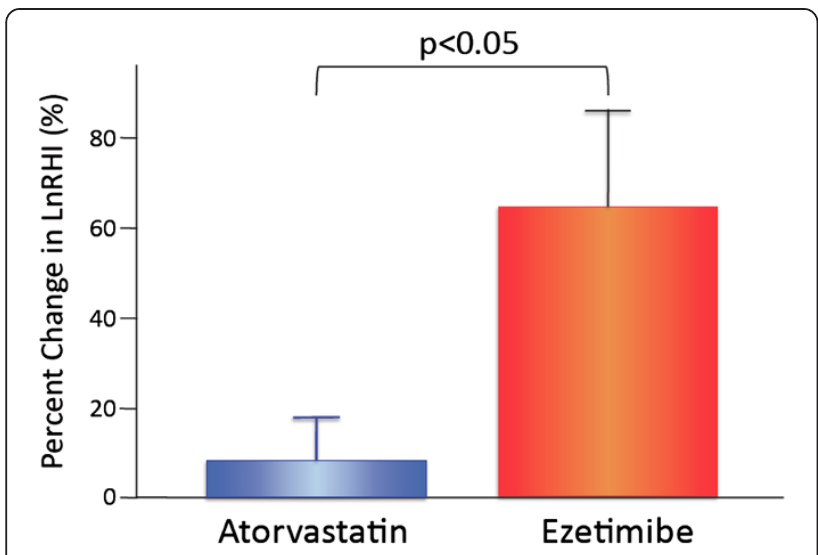

Figure 3 Percent changes in $\mathrm{LnRH}$ with atorvastatin and ezetimibe therapies. Percent changes in $\mathrm{LnRH}=$ (post-LnRHI - pre-LnRHI) $\times 100$ / pre-LnRHI.Bar graphs represent data of mean and standard error of mean. Atorvastatin group $(n=16)$ and ezetimibe group $(n=17)$. LnRHI: natural logarithmic transformed value of reactive hyperemia peripheral arterial tonometry index.

cholesterol-lowering therapy allocation revealed that ezetimibe therapy significantly correlated with improvements in endothelial function (Table 3, odds ratio: 6.42 , 95\% confidence interval: $1.13-36.47, \mathrm{p}=$ 0.036). The Hosmer-Lemeshow statistic was appropriate $(\mathrm{p}=0.53)$.

\section{Correlation between percent changes in LnRHI and various lipid parameters}

To determine the associated factors between the LDLlowering therapy-induced improvement in microvascular endothelial function and changes in lipid parameters, we investigated the correlation coefficient between percent changes in LnRHI and percent changes in plasma lipid variables during therapy. As shown in Table 4, percent changes in LnRHI did not significantly correlate with percent changes in total cholesterol, LDL, HDL, and triglyceride. Changes in LnRHI showed significant correlations with changes in cholesterol absorption or synthesis parameters during therapy (Table 4). The indicator of cholesterol synthesis and absorption balance (the ratio of lathosterol to campesterol) demonstrated the greatest correlation efficient value $(\mathrm{r}=0.459, \mathrm{p}=0.008)$ among all parameters. In the intra-group analyses, none of the parameters were significantly correlated with percent changes in LnRHI in the ezetimibe and atorvastatin groups (Tables 5 and 6).

\section{Discussion}

This is the first study to directly investigate the effectiveness of two different LDL-lowering strategies, cholesterol absorption inhibition and cholesterol synthesis suppression, on microvascular endothelial function in type $2 \mathrm{DM}$. We observed that ezetimibe monotherapy, when compared with atorvastatin monotherapy, significantly decreased

Table 3 Logistic regression analysis of baseline parameters for the improvement of LnRHI (>40\%)

\begin{tabular}{|c|c|c|c|c|c|c|}
\hline \multirow[t]{2}{*}{ Baseline variable } & \multicolumn{3}{|c|}{ Univariate regression } & \multicolumn{3}{|c|}{ Multivariate regression using forced inclusion model } \\
\hline & OR & $95 \% \mathrm{Cl}$ & p & OR & $95 \% \mathrm{Cl}$ & $\mathrm{p}$ \\
\hline Age (per year) & 1.02 & $0.94-1.11$ & 0.58 & 1.05 & $0.95-1.15$ & 0.33 \\
\hline Gender (male) & 2.28 & $0.54-9.67$ & 0.27 & 4.22 & $0.71-25.03$ & 0.11 \\
\hline Body mass index (per 1.0) & 1.06 & $0.83-1.36$ & 0.66 & - & - & - \\
\hline Hemoglobin A1c (per 0.1; \%) & 0.98 & $0.89-1.04$ & 0.33 & - & - & - \\
\hline Fasting plasma glucose (per 1.0; mg/dl) & 0.98 & $0.94-1.01$ & 0.17 & - & - & - \\
\hline Total cholesterol (per 1.0; mg/dl) & 0.99 & $0.97-1.03$ & 0.96 & - & - & - \\
\hline LDL cholesterol (per 1.0; mg/dl) & 0.98 & $0.95-1.01$ & 0.21 & - & - & - \\
\hline HDL cholesterol (per 1.0; mg/dl) & 1.01 & $0.96-1.06$ & 0.72 & - & - & - \\
\hline Triglyceride (per 1.0; mg/dl) & 1.01 & $0.99-1.02$ & 0.20 & - & - & - \\
\hline Sulfonylureas (yes) & 1.42 & $0.26-7.76$ & 0.69 & - & - & - \\
\hline Metformin (yes) & 1.60 & $0.34-7.65$ & 0.56 & - & - & - \\
\hline Alpha-glucosidase inhibitor (yes) & 0.54 & $0.11-2.62$ & 0.45 & - & - & - \\
\hline Thiazolidinedione (yes) & 0.86 & $0.07-1.66$ & 0.91 & - & - & - \\
\hline Glinide (yes) & 1.00 & $0.00--$ & 1.00 & - & - & - \\
\hline DPP4 inhibitor (yes) & 0.86 & $0.07-10.66$ & 0.91 & - & - & - \\
\hline Insulin (yes) & 1.74 & $0.40-7.91$ & 0.45 & - & - & - \\
\hline Ezetimibe-therapy (yes) & 4.88 & $1.01-23.57$ & 0.049 & 6.42 & $1.13-36.47$ & 0.036 \\
\hline
\end{tabular}

LnRHI: natural logarithmic transformed value of reactive hyperemia peripheral tonometry index; OR, odds ratio; $\mathrm{Cl}$, confidence interval; LDL, low-density lipoprotein; $\mathrm{HDL}$, high-density lipoprotein, DPP-4, dipeptidyl peptidase-4; Hosmer-Lemeshow $p=0.53$ in multivariate analysis. 
Table 4 Correlation between percent changes in lipid parameters and percent changes in LnRHI in all subjects

\begin{tabular}{lll}
\hline & $\mathbf{r}$ & $\mathbf{p}$-value \\
\hline Percent change in total cholesterol & -0.053 & 0.769 \\
Percent change in LDL cholesterol & -0.053 & 0.770 \\
Percent change in HDL cholesterol & -0.222 & 0.215 \\
Percent change in triglyceride & 0.236 & 0.186 \\
Percent change in NEFA & -0.115 & 0.524 \\
Percent change in lathosterol & 0.354 & 0.047 \\
Percent change in campesterol & -0.399 & 0.024 \\
Percent change in lathosterol/campesterol & 0.459 & 0.008 \\
\hline
\end{tabular}

LDL: low-density lipoprotein, HDL: high-density lipoprotein, NEFA: nonesterified fatty acid, LnRHI: natural logarithmic transformed value of reactive hyperemia peripheral tonometry index.

serum NEFA levels and significantly improved peripheral microvascular endothelial function using RH-PAT tests in stable patients with type $2 \mathrm{DM}$.

In patients with type $2 \mathrm{DM}$, lowering cholesterol using statins is clinically effective and is an established treatment for preventing cardiovascular events [3]. However, there are no clinical studies comparing the effects of LDL-lowering monotherapies, such as cholesterol synthesis suppression using statins or cholesterol absorption inhibition using ezetimibe, on endothelial function in patients with type $2 \mathrm{DM}$.

In the small intestine, cholesterol is absorbed through NPC1L1, the target molecule of ezetimibe [2]. Ezetimibe exhibits its LDL-lowering properties by blocking NPC1L1 in the small intestine without attenuating the mevalonate pathway [2]. Recently, it has been reported that inactivating mutations in the NPC1L1 gene provided significant protection against coronary heart disease [15]. It has also been reported that intestinal expression of NPC1L1 increased in patients with type 2 DM [16]. Additionally, the LDL-lowering effect of ezetimibe was significantly greater in DM [17], suggesting

Table 5 Correlation between percent changes in lipid parameters and in LnRHI in subjects treated by ezetimibe

\begin{tabular}{lll}
\hline & $\mathbf{r}$ & $\mathbf{p}$-value \\
\hline Percent change in total cholesterol & -0.246 & 0.336 \\
Percent change in LDL cholesterol & -0.375 & 0.138 \\
Percent change in HDL cholesterol & -0.220 & 0.397 \\
Percent change in triglyceride & 0.150 & 0.565 \\
Percent change in NEFA & 0.137 & 0.601 \\
Percent change in lathosterol & 0.283 & 0.289 \\
Percent change in campesterol & -0.273 & 0.306 \\
Percent change in lathosterol/campesterol & 0.324 & 0.221 \\
\hline
\end{tabular}

LDL: low-density lipoprotein, HDL: high-density lipoprotein, NEFA: nonesterified fatty acid, LnRHI: natural logarithmic transformed value of reactive hyperemia peripheral tonometry index.
Table 6 Correlation between percent changes in lipid parameters and in LnRHI in subjects treated by atorvastatin

\begin{tabular}{lll}
\hline & $\mathbf{r}$ & p-value \\
\hline Percent change in total cholesterol & -0.106 & 0.696 \\
Percent change in LDL cholesterol & -0.420 & 0.105 \\
Percent change in HDL cholesterol & 0.276 & 0.300 \\
Percent change in triglyceride & 0.438 & 0.065 \\
Percent change in NEFA & -0.055 & 0.893 \\
Percent change in lathosterol & -0.180 & 0.504 \\
Percent change in campesterol & -0.098 & 0.723 \\
Percent change in lathosterol/campesterol & -0.121 & 0.655 \\
\hline
\end{tabular}

LDL: low-density lipoprotein, HDL: high-density lipoprotein, NEFA: nonesterified fatty acid, LnRHI: natural logarithmic transformed value of reactive hyperemia peripheral tonometry index.

the potentially beneficial role of ezetimibe as a NPC1L1 inhibitor in the treatment of DM with dyslipidemia and vascular dysfunction $[8,9]$.

In the present study, each monotherapy resulted in a significant decrease in blood LDL, but the marker of the cholesterol synthesis/absorption balance showed a markedly different effect. We confirmed that the two treatments had opposite effects on endogenous metabolism of cholesterol synthesis/absorption, and found that changes in the cholesterol synthesis/absorption balance significantly correlated with changes in microvascular endothelial function in patients with DM. Interestingly, the effect of each monotherapy on peripheral microvascular endothelial function in patients with DM varied significantly. Ezetimibe monotherapy but not atorvastatin monotherapy significantly improved peripheral microvascular endothelial function. Effective cardiovascular actions through the inhibition of cholesterol absorption by ezetimibe would be clinically expected in DM [9].

Previously, Nochioka et al. reported that ezetimibe, when compared with pravastatin, significantly improved endothelial function as assessed by flow-mediated dilation (FMD) in young healthy volunteers [18]. The researchers identified a significant correlation between the reduction of a cholesterol absorption marker and improvement in FMD. In outpatients with type $2 \mathrm{DM}$, we found similar clinical results in the present study. Recently, the effect of pravastatin or ezetimibe on endothelial function was reported as evaluated by FMD in non-DM patients with hypercholesterolemia [19]. The researchers concluded that LDL-lowering by pravastatin or ezetimibe induced similar effects on vascular endothelial function improvement, and they suggested that the pleiotropic effects of statins were not important for improvement in vascular endothelial function. Although the LDL decrease was equal in the 
two treatment strategies in that study, the improvement in FMD tended to be greater with ezetimibe than with pravastatin (FMD change: ezetimibe $5.2 \% v s$. pravastatin $3.8 \%$ ), indicating the potentially beneficial use of ezetimibe. The major differences between that study and the present study are the baseline clinical backgrounds of patients (non-DM vs. DM and hypercholesterolemia [LDL: $180 \mathrm{mg} / \mathrm{dL}$ ] vs. mildly elevated cholesterol [LDL: $142 \mathrm{mg} / \mathrm{dL}$ ]), treatment period (6 weeks vs. 6 months), and the method of assessing endothelial function (FMD vs. RH-PAT). Several previous studies failed to demonstrate the beneficial effect of ezetimibe monotherapy on endothelial function [20-22]. These studies compared the effects of statin or ezetimibe on endothelial function in patients with heart failure $[20,21]$ or coronary artery disease with a small number of patients with DM [22], and the authors mentioned the 'pleiotropic effect of statin' for the treatment of cardiovascular disease. In contrast, Yunoki et al. reported the improvement achieved by ezetimibe on endothelial dysfunction in the postprandial phase [23] and in CAD patients with hypertriglyceridemia [24]. Moreover, Yamaoka-Tojo et al. reported the benefit of the add-on therapy of ezetimibe to statin therapy in patients at high risk of cardio-metabolic disease [25].

In the present study, atorvastatin did not significantly improve microvascular endothelial function in DM, although there was a greater reduction in LDL. The reason why atorvastatin did not improve endothelial function is unclear. Several clinical studies reported that statins could improve endothelial function in patients with DM [26-28]. In contrast, several randomized trials showed that statins did not improve endothelial function in patients with type 2 DM [29-32]. Recently, Zhang et al. reported a meta-analysis of placebo randomized studies using FMD as the measure of endothelial function to determine the effects of statins on endothelial function in patients with DM [33]. They found overall beneficial effects of statins on endothelial function in patients with DM but the beneficial effects were not observed in patients with type $2 \mathrm{DM}$, obesity (BMI $>27)$, and older age ( $>55$ years) in the sub-analysis. In the present study, all of the enrolled patients had type 2 DM and average age of 64.6 years. Thus, we speculated that our patients could be less responsive to statin therapy in terms of endothelial function improvement.

In the present study, we investigated the effects of ezetimibe and atorvastatin on the peripheral microvascular endothelial function assessed by RH-PAT. We focused mainly on the microcirculation rather than the large conduit arteries, such as the brachial artery that was used for the FMD measurement. Regarding the effect of statins on microvascular endothelial function, previous studies reported that there was no significant benefit of statin therapy in patients with DM [34-37]. This study is the first to report the improvement of microvascular endothelial dysfunction by ezetimibe in patients with type 2 DM.

Because LDL-lowering therapy is essential and effective for patients with DM in the clinical practice, we cannot conduct a placebo-controlled study with statin therapy for DM. We think that the results of the present study will shed light on the controversial subject of endothelial dysfunction therapy in patients with DM. Further studies evaluating larger numbers of DM cases are required to confirm the results of the present study.

The clinical properties of ezetimibe for the treatment of dyslipidemia are improvements in both hypercholesterolemia and postprandial dyslipidemia $[2,38]$. In the present study, we identified, for the first time, that ezetimibe significantly reduced NEFA in patients with DM. It has been reported that ezetimibe can significantly reduce fatty acid absorption in animal models [39], that NEFA is involved in the pathological conditions of insulin resistance [40], and that NEFA is associated with inflammation $[41,42]$ and endothelial dysfunction [43]. These findings suggest that NEFA could have great significance in the pathological conditions of DM [44]. In the present study, ezetimibe significantly reduced NEFA in patients with DM, but statin did not have the similar effect. Changes in NEFA might bring about different effects on the longterm metabolic balance of lipids and glucose [43]. A potential therapy to significantly reduce NEFA in DM has not been reported until now, and we think that this result has important clinical meaning. The impact of ezetimibemediated changes in NEFA on vascular endothelial function requires further detailed examination.

Both ezetimibe and atorvastatin therapy resulted in significant reductions of LDL; however, ezetimibe significantly improved endothelial function compared to atorvastatin. This finding suggests that ezetimibe added on to the LDLlowering effects by an unknown mechanism, and this unique property might contribute to the improvement of endothelial function in patients with DM. It has been reported that a postprandial metabolic disorder is deeply involved in endothelial dysfunction in DM, and ezetimibe improved postprandial hyperlipemia and its induced endothelial dysfunction [23]. We did not measure postprandial lipid parameters in the present study, but we can speculate that the ezetimibe-mediated improvement in postprandial dyslipidemia may potentially contribute to the beneficial vascular function. In a future study, we need to investigate the correlation between ezetimibe-mediated improvement of postprandial dyslipidemia and endothelial function in patients with DM.

Recent meta-analysis demonstrated that the statinmediated increase in HDL might not be significantly 
associated with the decrease in future cardiovascular events [45]. Regarding HDL, it has been reported that not only plasma quantity of HDL, but also functional quality of HDL is important for exerting vascular protective and anti-atherosclerotic effects [46]. In the present study, the HDL increase by atorvastatin was not associated with improvement of endothelial function in patients with DM. It will be worth to determine whether ezetimibe could modulate HDL function in a future study. Based on the present results, we think that changes in HDL by LDL-lowering therapy cannot be good indicators of improvement of microvascular endothelial function in patients with DM.

This study had several limitations. The study was a single center study and the protocol was open-labeled. Only a small number of patients made up the study population, and the study period was short. We did not evaluate serum levels of postprandial lipids and oxysterol. As the study patients were stable and had relatively favorable and controlled type $2 \mathrm{DM}$, were not obese, and did not have cardiovascular diseases, further studies are required using a larger number of patients with DM with a broad spectrum of clinical backgrounds.

In conclusion, both ezetimibe monotherapy and atorvastatin monotherapy significantly reduced $\mathrm{LDL}$, and ezetimibe monotherapy significantly increased LnRHI in patients with DM. Ezetimibe, but not atorvastatin, significantly reduced serum levels of NEFA. In this population of patients with type $2 \mathrm{DM}$, ezetimibe monotherapy significantly ameliorated dyslipidemia and improved peripheral microvascular endothelial dysfunction.

\section{Abbreviations \\ BMI: Body mass index; DM: Diabetes mellitus; FPG: Fasting plasma glucose; FMD: Flow-mediated dilation; HbA1c: Hemoglobin A1c; HDL: High-density lipoprotein cholesterol; LDL: Low-density lipoprotein cholesterol; $\mathrm{RH}$ - PAT: Reactive hyperemia peripheral arterial tonometry; LnRHI: Natural logarithmic transformed value for the RH-PAT index; NPC1L1: Niemann-Pick C1-Like 1; NEFA: Non-esterified free fatty acid; RHI: RH-PAT index.}

\section{Competing interests}

Dr. Hideaki Jinnouchi received Consultation from Sanofi U.S., and Novo Nordisk Inc.; Research support from Eli Lilly Japan K.K., Novo Nordisk Inc., Boehringer Ingelheim Pharmaceuticals, Inc., Sumitomo Dainippon Pharma Co., Ltd., GlaxoSmithKline, Takeda Pharmaceutical Co., Ltd. Daiichi-Sankyo Co., Ltd., Taisho Pharmaceutical Co., Ltd., Astellas Pharma US, Inc., and AstraZeneca Pharmaceuticals LP; Speaker's Bureau from Sanofi U.S., Eli Lilly Japan K.K., Takeda Pharmaceutical Co., Ltd., Astellas Pharma US, Inc., Mitsubishi Tanabe Pharma Corp., Daiichi-Sankyo Co., Ltd., Boehringer Ingelheim Pharmaceuticals, Inc., Kissei Pharmaceutical Co., Ltd., Kowa Pharmaceuticals, Novartis Pharmaceuticals Corp., AstraZeneca Pharmaceuticals LP, MSD, Inc., and Mochida Pharmaceutical Co., Ltd. All other authors declare that they have no competing interests.

\section{Authors' contributions}

SS and HJ conceived and designed the study, acquired data, or analyzed and interpreted data. SS, HJ, KM, KH, TS, NK, FM, KK, and TJ drafted the manuscript or revised it critically for important intellectual content. SS, HJ, $\mathrm{KM}, \mathrm{KH}, \mathrm{TS}, \mathrm{NK}, \mathrm{FM}, \mathrm{KK}$, and TJ approved the final version to be published. SS, $H J, K M, K H, T S, N K, F M, K K$, and TJ agree to be accountable for all aspects of the work SS, HJ, and KM conducted statistical analyses and reviewed/edited the manuscript. All authors read and approved the final manuscript.

\section{Acknowledgement}

The authors thank Noriko Nakamura for her skillful technical assistance.

\section{Author details}

'Diabetes Care Center, Jinnouchi Hospital, 6-2-3 Kuhonji, Chuo-ku, Kumamoto 862-0976, Japan. ${ }^{2}$ Diabetes Care Center, Cardiovascular Division, Jinnouchi Hospital, 6-2-3 Kuhonji, Chuo-ku, Kumamoto 862-0976, Japan. ${ }^{3}$ Department of Cardiovascular Medicine, Faculty of Life Sciences, Graduate School of Medical Science, Kumamoto University, 1-1-1 Honjo, Chuo-ku, Kumamoto 862-8556, Japan. ${ }^{4}$ Division of Preventive Cardiology, Department of Cardiovascular Medicine, Kumamoto University Hospital, 1-1-1 Honjo, Chuo-ku, Kumamoto 862-8556, Japan. ${ }^{5}$ Department of Community Medicine, Kumamoto University Hospital, 1-1-1 Honjo, Chuo-ku, Kumamoto 862-8556, Japan.

Received: 31 January 2015 Accepted: 30 March 2015

Published online: 23 April 2015

\section{References}

1. Bays HE. Lowering low-density lipoprotein cholesterol levels in patients with type 2 diabetes mellitus. Int J Gen Med. 2014;7:355-64.

2. Phan BA, Dayspring TD, Toth PP. Ezetimibe therapy: mechanism of action and clinical update. Vasc Health Risk Manag. 2012;8:415-27.

3. Colhoun HM, Betteridge DJ, Durrington PN, Hitman GA, Neil HA, Livingstone SJ, et al. Primary prevention of cardiovascular disease with atorvastatin in type 2 diabetes in the Collaborative Atorvastatin Diabetes Study (CARDS): multicentre randomised placebo-controlled trial. Lancet. 2004;364(9435):685-96.

4. Swerdlow DI, Preiss D, Kuchenbaecker KB, Holmes MV, Engmann JE, Shah T, et al. HMG-coenzyme A reductase inhibition, type 2 diabetes, and bodyweight: evidence from genetic analysis and randomised trials. Lancet. 2014;385(9965):351-61.

5. Masuda D, Nakagawa-Toyama Y, Nakatani K, Inagaki M, Tsubakio-Yamamoto $\mathrm{K}$, Sandoval JC, et al. Ezetimibe improves postprandial hyperlipidaemia in patients with type Ilb hyperlipidaemia. Eur J Clin Invest. 2009;39(8):689-98.

6. Nozue T, Michishita I, Mizuguchi I. Effects of ezetimibe on glucose metabolism in patients with type 2 diabetes: A 12-week, open-label, uncontrolled, pilot study. Curr Ther Res Clin Exp. 2010;71 (4):252-8.

7. Ichimori S, Shimoda S, Goto R, Matsuo Y, Maeda T, Furukawa N, et al. Ezetimibe improves glucose metabolism by ameliorating hepatic function in Japanese patients with type 2 diabetes. J Diabetes Investig. 2012;3(2):179-84.

8. Sarigianni M, Katsiki N, Mikhailidis DP. Ezetimibe in diabetes: more than cholesterol lowering? Curr Med Res Opin. 2010;26(10):2517-20.

9. Hayek S, Canepa Escaro F, Sattar A, Gamalski S, Wells KE, Divine G, et al. Effect of ezetimibe on major atherosclerotic disease events and all-cause mortality. Am J Cardiol. 2013;111(4):532-9.

10. Kuvin JT, Patel AR, Sliney KA, Pandian NG, Sheffy J, Schnall RP, et al. Assessment of peripheral vascular endothelial function with finger arterial pulse wave amplitude. Am Heart J. 2003;146(1):168-74.

11. Matsuzawa Y, Sugiyama S, Sugamura K, Nozaki T, Ohba K, Konishi M, et al. Digital assessment of endothelial function and ischemic heart disease in women. J Am Coll Cardiol. 2010;55(16):1688-96.

12. Matsuzawa $Y$, Sugiyama $S$, Sumida $H$, Sugamura $K$, Nozaki T, Ohba $K$, et al. Peripheral endothelial function and cardiovascular events in high-risk patients. J Am Heart Assoc. 2013;2(6):e000426.

13. McCrea CE, Skulas-Ray AC, Chow M, West SG. Test-retest reliability of pulse amplitude tonometry measures of vascular endothelial function: implications for clinical trial design. Vasc Med. 2012;17(1):29-36.

14. Akiyama E, Sugiyama S, Matsuzawa Y, Konishi M, Suzuki H, Nozaki T, et al. Incremental prognostic significance of peripheral endothelial dysfunction in patients with heart failure with normal left ventricular ejection fraction. J Am Coll Cardiol. 2012;60(18):1778-86.

15. Stitziel NO, Won HH, Morrison AC, Peloso GM, Do R, Lange LA, et al. Inactivating mutations in NPC1L1 and protection from coronary heart disease. N Engl J Med. 2014;371(22):2072-82.

16. Lally SE, Owens D, Tomkin GH. Sitosterol and cholesterol in chylomicrons of type 2 diabetic and non-diabetic subjects: the relationship with ATP binding 
cassette proteins G5 and G8 and Niemann-Pick C1-like 1 mRNA. Diabetologia. 2007:50(1):217-9.

17. Okada K, Yagyu H, Kotani K, Miyamoto M, Osuga J, Nagasaka S, et al. Lipidlowering effects of ezetimibe for hypercholesterolemic patients with and without type 2 diabetes mellitus. Endocr J. 2010;57(10):903-8.

18. Nochioka K, Tanaka S, Miura M, Zhulanqiqige do E, Fukumoto Y, Shiba $\mathrm{N}$. Ezetimibe improves endothelial function and inhibits Rho-kinase activity associated with inhibition of cholesterol absorption in humans. Circ J. 2012;76(8):2023-30.

19. Grigore L, Raselli S, Garlaschelli K, Redaelli L, Norata GD, Pirillo A, et al. Effect of treatment with pravastatin or ezetimibe on endothelial function in patients with moderate hypercholesterolemia. Eur J Clin Pharmacol. 2013;69(3):341-6.

20. Landmesser U, Bahlmann F, Mueller M, Spiekermann S, Kirchhoff N, Schulz S, et al. Simvastatin versus ezetimibe: pleiotropic and lipid-lowering effects on endothelial function in humans. Circulation. 2005;111(18):2356-63.

21. Gounari $P$, Tousoulis D, Antoniades C, Kampoli AM, Stougiannos $P$, Papageorgiou N, et al. Rosuvastatin but not ezetimibe improves endothelial function in patients with heart failure, by mechanisms independent of lipid lowering. Int J Cardiol. 2010;142(1):87-91.

22. Fichtlscherer S, Schmidt-Lucke C, Bojunga S, Rossig L, Heeschen C, Dimmeler S, et al. Differential effects of short-term lipid lowering with ezetimibe and statins on endothelial function in patients with CAD: clinical evidence for "pleiotropic" functions of statin therapy. Eur Heart J. 2006;27(10):1182-90.

23. Yunoki K, Nakamura K, Miyoshi T, Enko K, Kohno K, Morita H, et al. Ezetimibe improves postprandial hyperlipemia and its induced endothelial dysfunction. Atherosclerosis. 2011;217(2):486-91.

24. Yunoki K, Nakamura K, Miyoshi T, Enko K, Kubo M, Murakami M, et al. Impact of hypertriglyceridemia on endothelial dysfunction during statin +/ezetimibe therapy in patients with coronary heart disease. Am J Cardiol. 2011;108(3):333-9.

25. Yamaoka-Tojo M, Tojo T, Kosugi R, Hatakeyama Y, Yoshida Y, Machida Y, et al. Effects of ezetimibe add-on therapy for high-risk patients with dyslipidemia. Lipids Health Dis. 2009;8:41.

26. Economides PA, Caselli A, Tiani E, Khaodhiar L, Horton ES, Veves A. The effects of atorvastatin on endothelial function in diabetic patients and subjects at risk for type 2 diabetes. J Clin Endocrinol Metab. 2004;89(2):740-7.

27. Koh KK, Quon MJ, Han SH, Ahn JY, Jin DK, Kim HS, et al. Vascular and metabolic effects of combined therapy with ramipril and simvastatin in patients with type 2 diabetes. Hypertension. 2005;45(6):1088-93.

28. Ceriello A, Assaloni R, Da Ros R, Maier A, Piconi L, Quagliaro L, et al. Effect of atorvastatin and irbesartan, alone and in combination, on postprandial endothelial dysfunction, oxidative stress, and inflammation in type 2 diabetic patients. Circulation. 2005;111(19):2518-24.

29. Balletshofer BM, Goebbel S, Rittig K, Enderle M, Schmolzer I, Wascher TC, et al. Intense cholesterol lowering therapy with a $\mathrm{HMG}-\mathrm{COA}$ reductase inhibitor does not improve nitric oxide dependent endothelial function in type-2-diabetes-a multicenter, randomised, double-blind, three-arm placebo-controlled clinical trial. Exp Clin Endocrinol Diabetes. 2005;113(6):324-30.

30. Beishuizen ED, Tamsma JT, Jukema JW, van de Ree MA, van der Vijver JC, Meinders $A E$, et al. The effect of statin therapy on endothelial function in type 2 diabetes without manifest cardiovascular disease. Diabetes Care. 2005;28(7):1668-74.

31. van Venrooij FV, van de Ree MA, Bots ML, Stolk RP, Huisman MV, Banga JD Aggressive lipid lowering does not improve endothelial function in type 2 diabetes: the Diabetes Atorvastatin Lipid Intervention (DALI) Study: a randomized, double-blind, placebo-controlled trial. Diabetes Care. 2002;25(7):1211-6.

32. Tantikosoom W, Thinkhamrop B, Kiatchusakul S, Jarernsiripornkul N, Srinakarin J, Ojongpian S. Randomized trial of atorvastatin in improving endothelial function in diabetics without prior coronary disease and having average cholesterol level. J Med Assoc Thai. 2005;88(3):399-406.

33. Zhang L, Gong D, Li S, Zhou X. Meta-analysis of the effects of statin therapy on endothelial function in patients with diabetes mellitus. Atherosclerosis. 2012;223(1):78-85.

34. Fegan PG, Shore AC, Mawson D, Tooke JE, MacLeod KM. Microvascular endothelial function in subjects with Type 2 diabetes and the effect of lipidlowering therapy. Diabet Med. 2005;22(12):1670-6.

35. Prazny M, Kasalova Z, Mazoch J, Kvasnicka J, Skrha J. Microvascular reactivity and endothelial function in type 2 diabetic patients with hyperlipidemia treated with simvastatin: 3-year follow-up. Prague Med Rep. 2009;110(4):290-300.

36. van de Ree MA, Huisman MV, de Man FH, van der Vijver JC, Meinders AE, Blauw GJ. Impaired endothelium-dependent vasodilation in type 2 diabetes mellitus and the lack of effect of simvastatin. Cardiovasc Res. 2001;52(2):299-305.

37. Mansourati J, Newman LG, Roman SH, Travis A, Rafey M, Phillips RA. Lipid lowering does not improve endothelial function in subjects with poorly controlled diabetes. Diabetes Care. 2001;24(12):2152-3.

38. Sandoval JC, Nakagawa-Toyama Y, Masuda D, Tochino Y, Nakaoka H, Kawase $\mathrm{R}$, et al. Molecular mechanisms of ezetimibe-induced attenuation of postprandial hypertriglyceridemia. J Atheroscler Thromb. 2010;17(9):914-24

39. Labonte ED, Camarota LM, Rojas JC, Jandacek RJ, Gilham DE, Davies JP, et al. Reduced absorption of saturated fatty acids and resistance to diet-induced obesity and diabetes by ezetimibe-treated and Npc111-/mice. Am J Physiol Gastrointest Liver Physiol. 2008;295(4):G776-83.

40. Boden G. Obesity, insulin resistance and free fatty acids. Curr Opin Endocrinol Diabetes Obes. 2011;18(2):139-43.

41. Kennedy A, Martinez K, Chuang CC, LaPoint K, McIntosh M. Saturated fatty acid-mediated inflammation and insulin resistance in adipose tissue: mechanisms of action and implications. J Nutr. 2009;139(1):1-4.

42. Legrand-Poels S, Esser N, L'Homme L, Scheen A, Paquot N, Piette J. Free fatty acids as modulators of the NLRP3 inflammasome in obesity/type 2 diabetes. Biochem Pharmacol. 2014;92(1):131-41.

43. Wyne KL. Free fatty acids and type 2 diabetes mellitus. Am J Med. 2003:115(Suppl 8A):29S-36.

44. Zhang H, Dellsperger KC, Zhang C. The link between metabolic abnormalities and endothelial dysfunction in type 2 diabetes: an update. Basic Res Cardiol. 2012;107(1):237.

45. Boekholdt SM, Arsenault BJ, Hovingh GK, Mora S, Pedersen TR, Larosa JC, et al. Levels and changes of HDL cholesterol and apolipoprotein A-I in relation to risk of cardiovascular events among statin-treated patients: a meta-analysis. Circulation. 2013;128(14):1504-12.

46. Rohatgi A, Khera A, Berry JD, Givens EG, Ayers CR, Wedin KE, et al. HDL cholesterol efflux capacity and incident cardiovascular events. N Engl J Med. 2014;371(25):2383-93.

\section{Submit your next manuscript to BioMed Central and take full advantage of:}

- Convenient online submission

- Thorough peer review

- No space constraints or color figure charges

- Immediate publication on acceptance

- Inclusion in PubMed, CAS, Scopus and Google Scholar

- Research which is freely available for redistribution

Submit your manuscript at www.biomedcentral.com/submit
C BioMed Central 